\title{
Ionic Liquid-decorated Copolymer Binders for Silicon/Graphite Anodes with Enhanced Rate Capability and Excellent Cycle Stability
}

Xiaokai Li, Heng Chen, ${ }^{*}$ Ming Chen, Jiawei Qi, Shaojun Chen, Haitao Zhuo*

College of Chemistry and Environmental Engineering, Shenzhen University, Shenzhen, 518060, China

Corresponding authors.

E-mail: polyhengchen@szu.edu.cn (H. Chen) \& haitaozhuo@163.com (H. Zhuo)

Table S1. Tensile test results of various polymer binders.

\begin{tabular}{cccc}
\hline Sample & $\boldsymbol{\sigma}_{\mathbf{T}}{ }^{\mathbf{a}}(\mathbf{M P a})$ & ${\boldsymbol{\boldsymbol { \varepsilon } _ { \mathbf { T } }}}^{\mathbf{a}} \mathbf{( \% )}$ & $\left.\mathbf{E}_{\mathbf{T}}{ }^{\mathbf{a}} \mathbf{( M P a}\right)$ \\
\hline PVDF & $21.48 \pm 3.32$ & $395 \pm 61$ & $123.66 \pm 13.22$ \\
PAA & $1.05 \pm 0.21$ & $703 \pm 132$ & $8.85 \pm 0.65$ \\
CP0 & $0.76 \pm 0.15$ & $961 \pm 232$ & $2.37 \pm 0.14$ \\
CP1 & $1.16 \pm 0.09$ & $554 \pm 60$ & $4.41 \pm 0.30$ \\
CP5 & $1.26 \pm 0.10$ & $288 \pm 37$ & $5.80 \pm 0.26$ \\
\hline
\end{tabular}

${ }^{a} \sigma_{T}, \varepsilon_{T}$, and $E_{T}$ refer to the tensile strength, elongation at break, and Young's modulus, respectively. 

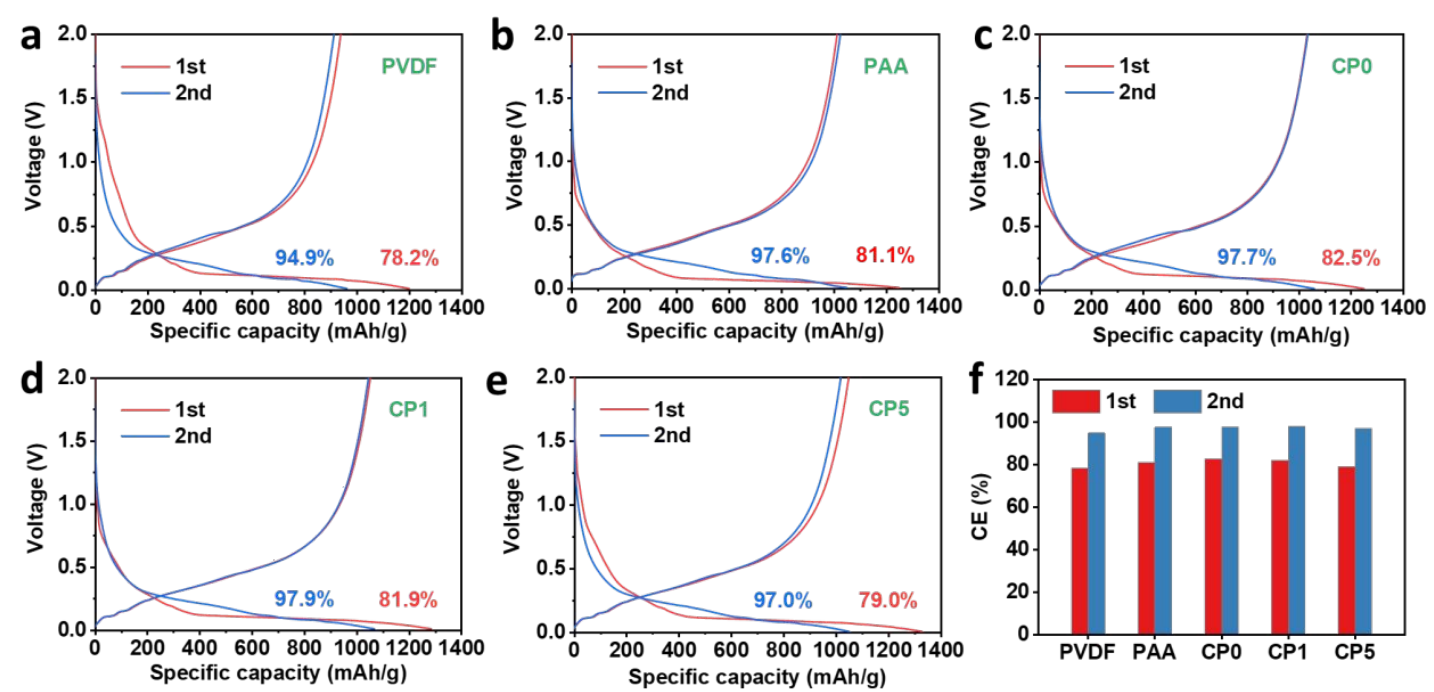

Figure S1. (a)-(e) Two initial charge/discharge curves of Si/Gr anodes obtained with different binders. (f) Coulombic efficiencies of two formation cycles using different binders.
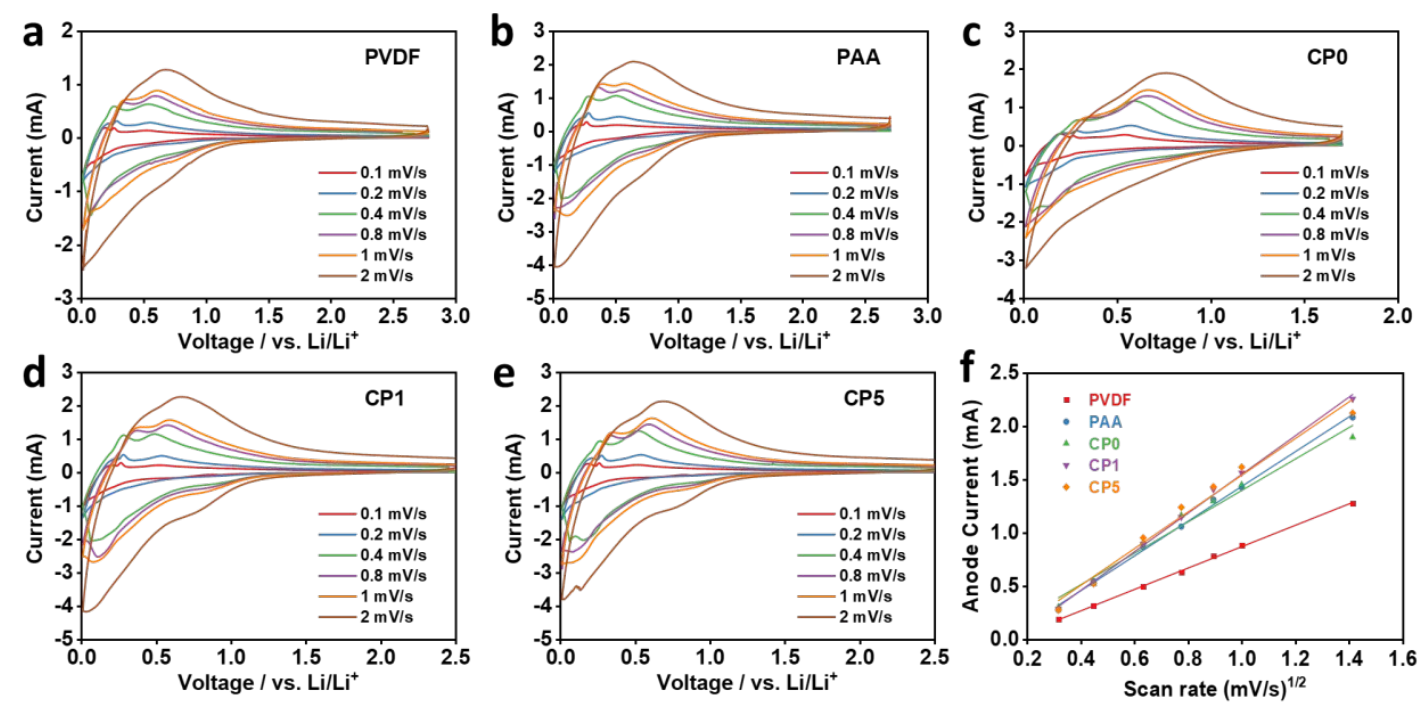

Figure S2. (a)-(e) CV curves of $\mathrm{Si} / \mathrm{Gr}$ anodes with various binders at different scan rates. (f) Plots of peak current $v s$ square root of scan rates for various binders obtained from the $\mathrm{CV}$. 


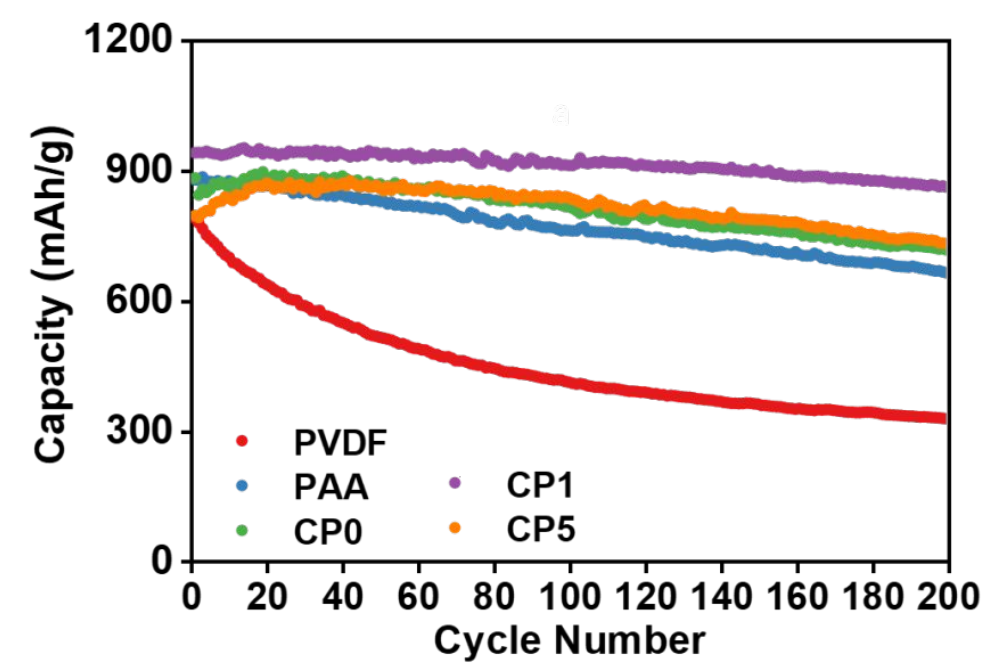

Figure S3. Specific discharge capacity for $\mathrm{Si} / \mathrm{Gr}$ anode with different binders in 200 cycles.

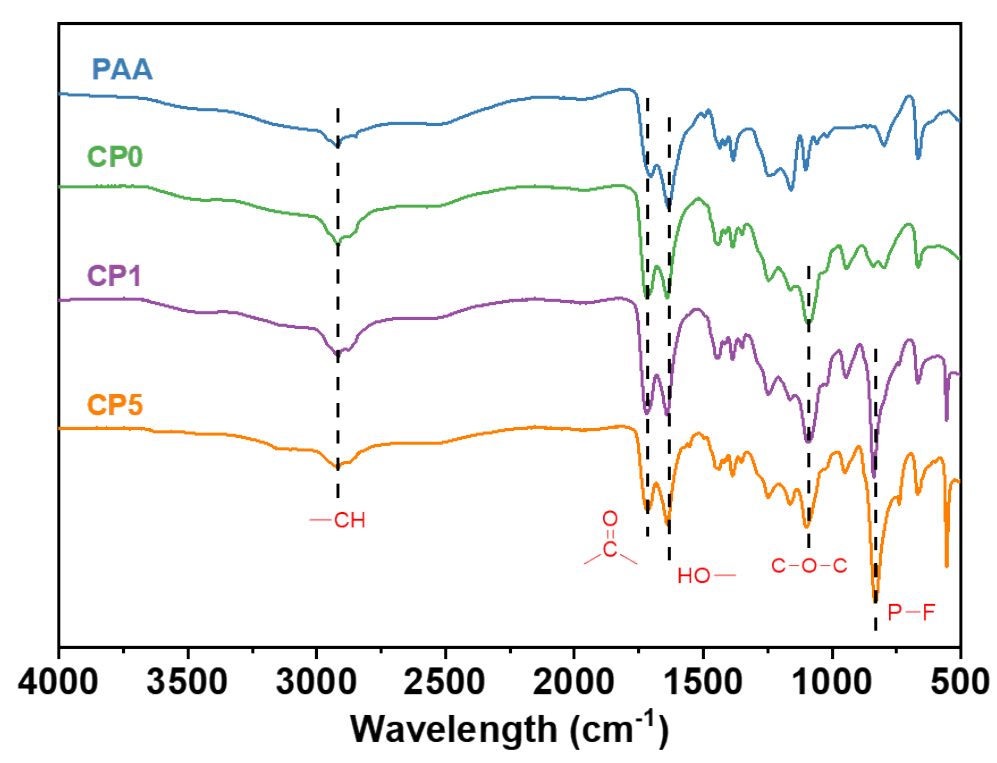

Figure S4. FTIR spectra of as-synthesized copolymer binders. 